\title{
Skin endometriosis at the caesarean section scar: A case report
}

\section{Beatriz Di Martino Ortiz¹, Osmar Manuel Cuenca Torres²}

\author{
${ }^{1}$ Dermatology Department, Clinicas Hospital, Faculty of Medical Sciences, National University of Ausunción-Paraguay, \\ ${ }^{2}$ Surgical Department, Clinicas Hospital, Faculty of Medical Sciences, National University of Ausunción-Paraguay \\ Corresponding author: Prof. Dr. Beatriz Di Martino Ortiz, E-mail: beatrizdimartino@gmail.com
}

\begin{abstract}
Endometriosis is a pathology little referenced in the dermatological literature and is defined as the presence of endometrial tissue outside the uterus. When it is a well-defined mass of endometriosis it is called endometrioma. Cutaneous endometriosis is one of the rare gynecological conditions. Cutaneous endometriosis is a disorder that primarily affects women of reproductive age. The disorder is most commonly associated with cyclical pain during menses, but it can be difficult to diagnose in the absence of these symptoms and requires biopsy testing for a definitive diagnosis. We report on a case of a 39-year-old patient who presented with pain at the cearean section scar. She was ultimately diagnosed with cutaneous endometriosis and underwent surgical excision.
\end{abstract}

Key words: Scar endometriosis; Cutaneous endometriosis

\section{INTRODUCTION}

Endometriosis represents the presence of nonneoplastic endometrial tissue outside the uterus. The disease is relatively common and typically affects the ovaries and presents with deep pelvic pain, dyspareunia, and dysmenorrhea.

The usually presentation location is usually the intrapelvic location and among them the most frequent ovarian form. However, extra-pelvic location may occur in more than $12 \%$ of women with endometriosis $[1,2]$ and affect any part of the body, even the skin [3].

Cutaneous endometriosis is relatively uncommon and occurs when endometrial glands and stroma reside in the skin.

Cutaneous endometriosis can be divided into primary and secondary endometriosis.

Endometriosis usually occurs in the form of diffuse involvement, in plaques, although sometimes it does so by adopting cystic or tumor morphology. When endometriosis occurs as a well-defined mass, it called endometrioma [4].

The pathogenesis for primary cutaneous endometriosis remains unclear, but secondary cutaneous endometriosis is believed to occur due to seeding after abdominal or pelvic surgery.

Because the condition is rare and can mimic presentations of other diseases, such as keloid or dermatofibroma, cutaneous endometriosis can be difficult to diagnose.

Punch biopsy can be performed to obtain tissue for histopathologic testing, but physicians must take care to obtain abdominal ultrasound if there is potential for uterocutaneous fistula. Once the diagnosis has been established, treatment options include hormonal agents and surgical excision with wide margins.

\section{CASE REPORT}

A 39-year-old patient with no family or personal history of interest, with a gynecological history of cesarean

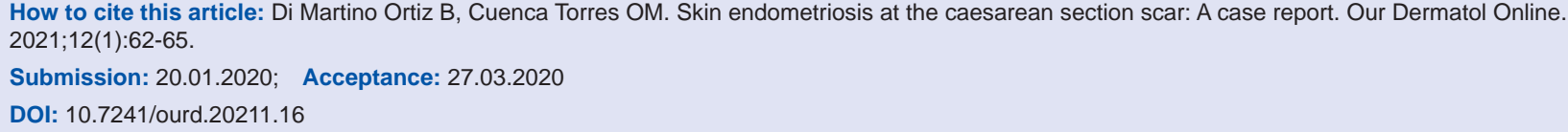


delivery 7 years ago without complications, without other valuable gynecological records.

Three years after the surgical history, she noticed a tumor of approximately $10 \mathrm{~mm}$ in diameter in the left lateral third of Pfannenstiel's scar. This tumor 1 year ago undergoes progressive growth, accompanied by stabbing pain related to the menstrual cycle, with local color change.

\section{Physical Examination}

A tumor of $50 \mathrm{~mm}$ in diameter, of elastic solid consistency, mobile that does not impress being adhered to deep planes, is observed.

According to the patient's clinic, the presumptive diagnosis of soft tissue endometriosis is made and it is decided to perform surgical excision with loco regional anesthesia.

Losangic incision is made on the tumor with a $20 \mathrm{~mm}$ window, being removed in block, it reaches an aponeurotic plane without exceeding it, a piece is sent to a pathological anatomy. There was a satisfactory evolution in the postoperative period.

\section{Pathological Anatomy}

A surgical piece that measures $55 \times 25 \times 45 \mathrm{~mm}$ of major axes, upholstered by a skin losange, is remitted.

The cut shows a poorly defined and non-encapsulated nodular formation of $20 \mathrm{~mm}$ of major axes. On the periphery of the same, multiple small cystic cavities full of hematic material are observed and in the center a yellow white tissue, of solid elastic consistency.

Serial cuts are made and the sample is processed routinely (Fig. 1).

At the histopathological examine there are multiple endometrial glands with surrounding endometrial stroma (Fig. 2). Decidualization of the stroma is not. Smooth muscle metaplasia is found. The glands show variable cystic dilatation and may contain blood and debris. There is not hemosiderin pigment There is dense fibrosis between the endometriotic foci.

\section{Final Diagnosis}

\section{Cutaneous endometriosis.}

(c) Our Dermatol Online 1.2021

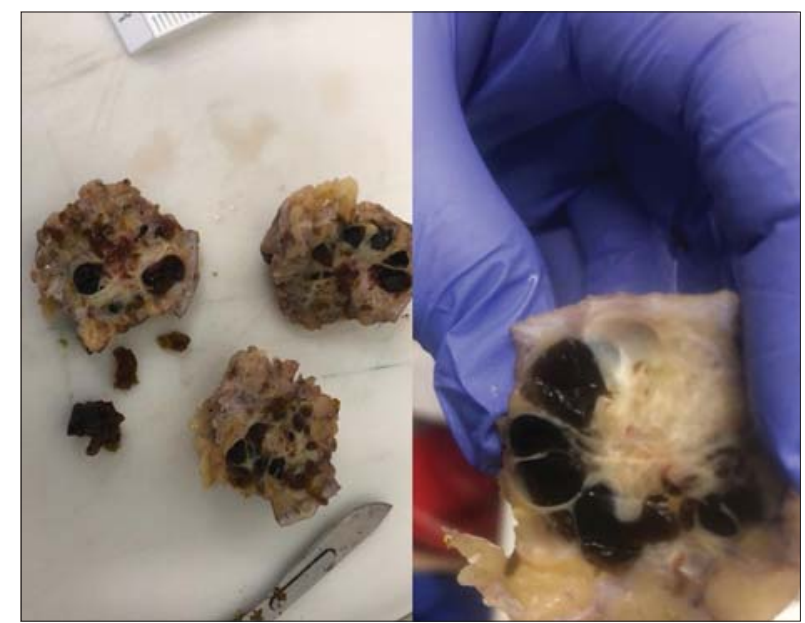

Figure 1: Gross pathology. The surface cut shows a poorly defined and non-encapsulated nodular formation of $20 \mathrm{~mm}$ of major axes with multiple small cystic cavities full of hematic material at the perifery.

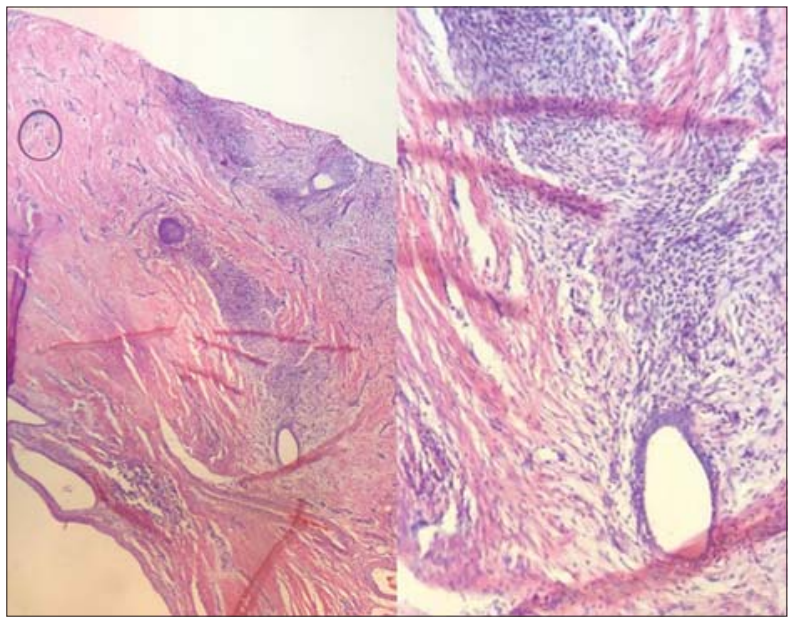

Figure 2: Histopathology. Endometrial glands with surrounding endometrial stroma (HE4X; HE20X).

\section{DISCUSSION}

Endometriosis is a chronic inflammatory reaction characterized by the presence of endometriomas outside the uterine cavity. It mainly causes painful symptoms and infertility while some women don't experience symptoms at all. The prevalence in the general female population is $2 \%$ to $10 \%$ but reaches up to $50 \%$ in infertile women [5].

The main etiology of endometriosis is not clear, but many studies suggest the hematogenous or lymphatic spread of stem cells from bone marrow or coelomic metaplasia [6].

Classically presents as a firm subcutaneous papule or nodule that averages $2 \mathrm{~cm}$ in diameter [7]. Its color can range from blue or violaceous to brown or skin- 
colored. Patients frequently experience cyclical pain, swelling, and even bleeding that corresponds with their menstrual cycle [8].

A diagnosis of cutaneous endometriosis can be made once the presence of endometrial glands and stroma in the skin is established. Notably, an ultrasound should be completed prior to performing a punch biopsy of the lesion to rule out the presence of uterocutaneous fistula.

The skin is an uncommon location for endometriosis, and cutaneous endometriosis cases comprise $<1 \%$ of all cases in large series (8). One review demonstrated that only 109 cases of cutaneous endometriosis had been described in the literature up to that point in time [9].

Cutaneous endometriosis is subdivided into two categories depending on patients' surgical history. Primary cutaneous endometriosis refers to cases in which the endometriosis develops spontaneously without any history of local surgery. It is the less common of the two (only $30 \%$ of patients present without a surgical history that could explain their cutaneous manifestations). Secondary cutaneous endometriosis, also called scar endometriosis, is associated with prior abdominal or pelvic surgery [7].

Our patient was diagnosed with cutaneous endometriosis based on the cyclical nature of her pain and the dermatopathologist diagnosis is in bad order. Identifying the patient's surgical history was crucial to subclassifying our patient's involvement as most likely being secondary in nature.

The most common location for both primary and secondary cutaneous endometriosis is the umbilicus. Umbilical cutaneous endometriosis comprises $30 \%$ to $40 \%$ of all cutaneous endometriosis cases, but other locations such as the groin, arm, episiotomy wounds, appendectomy scars, and cesarean scars have also been described [7].

Secondary cutaneous endometriosis is perhaps easier to conceptualize, and the prevailing hypothesis remains that endometrial cells dislodged during surgery seed the wound within and adjacent to the incision sites. In cases of primary cutaneous endometriosis, some postulate that seeding occurs hematogenously or via lymphatics [7].

Cutaneous endometriosis can mimic variable number of pathologies and also can mimic malignancy.
The most effective treatment of cutaneous endometriosis It is the surgical one. In the exeresis the focus of endometriosis with wide margins of $5-10 \mathrm{~mm}$ may be done with the intention of avoiding recurrence $[3,10,11]$.

The incidence of cancer on an ovarian endometriosis site is $1 \%$. The incidence of cancer in a focus of extrapelvic endometriosis is unknown [12]. Few cases of cancers of endometriosis on the abdominal wall have been described [13].

\section{Consent}

The examination of the patient was conducted according to the Declaration of Helsinki principles.

The authors certify that they have obtained all appropriate patient consent forms. In the form the patient(s) has/have given his/her/their consent for his/her/their images and other clinical information to be reported in the journal. The patients understand that their names and initials will not be published and due efforts will be made to conceal their identity, but anonymity cannot be guaranteed.

\section{REFERENCES}

1. Daisley Jr. D, Trim S, Daisley AR. Caesarean section scar endometriosis: A case report and review of the literature with special emphasis on malignant transformation. Our Dermatol Online. 2018;9:176-9.

2. Nellihela L, Al-Adnani M, Kufeji D. Primary umbilical endometriosis in an adolescent girl: unsuspected pathology. European J Pediatr Surg Rep. 2020;8:e10-e13.

3. Kocher M, Hardie A, Schaefer A, McLaren T, Kovacs M. Cesareansection scar endometrioma: a case report and review of the literature. J Radiol Case Rep. 2017;11:16-26.

4. Ozturk A, Kaya C, Bozkurtoglu H, Tan N, Yananli ZD, Ucmakli E. Scar endometrioma: an uncommon yet easily treated condition. J Reprod Med. 2016 May-Jun;61(5-6):249-53.

5. Dunselman GAJ, Vermeulen N, Becker C, Calhaz-Jorge C, D'Hooghe T, De Bie B, et al. ESHRE guideline: management of women with endometriosis. Hum Reprod. 2014;29:40012.

6. Mansouri S, Mai S, Hassam B, Meziane M. Omphalolith: a rare umbilical concretion. Our Dermatol Online. 2019;10:411-2.

7. Loh SH, Lew BL, Sim WY. Primary cutaneous endometriosis of umbilicus. Ann Dermatol. 2017;29:621-5.

8. Ao X, Xiong W, Tan SQ. Laparoscopic umbilical trocar port site endometriosis: A case report. World J Clin Cases. 2020;8:1532-7.

9. Lopez-Soto A, Sanchez-Zapata MI, Martinez-Cendan JP, Ortiz Reina S, Bernal Mañas CM, Remezal Solano M. Cutaneous endometriosis: Presentation of 33 cases and literature review. Eur J Obstet Gynecol Reprod Biol. 2018;221:58-63.

10. Batista M, Alves F, Cardoso J, Gonçalo M. Cutaneous endometriosis: a differential diagnosis of umbilical nodule. Acta Med Port. 2020;33:282-4.

11. Carsote M, Terzea DC, Valea A, Gheorghisan-Galateanu AA. Abdominal wall endometriosis (a narrative review). Int J Med Sci. 2020;17:536-42. 
www.odermatol.com

12. Lin KY, Chang CY, Lin WC, Wan L. Increased risk of endometriosis in patients with endometritis - a nationwide cohort study involving 84,150 individuals. Ginekol Pol. 2020;91:193-200.

13. Guo SW. Cancer-associated mutations in endometriosis: shedding light on the pathogenesis and pathophysiology. Hum Reprod Update. 2020;26:423-49.
Copyright by Beatriz Di Martino Ortiz, et al. This is an open access article distributed under the terms of the Creative Commons Attribution License, which permits unrestricted use, distribution, and reproduction in any medium, provided the original author and source are credited.

Source of Support: Nil, Conflict of Interest: None declared. 